\title{
Interdisciplinaridade em trabalhos na Conferência Nacional sobre Modelagem na Educação Matemática
}

\author{
Thaís Ribeiro da Silva \\ Paloma Silva de Lima \\ Bárbara de Paula Motta Mirson \\ Jussara de Loiola Araújo
}

\begin{abstract}
Resumo: Neste artigo, apresentamos resultados parciais de um estudo do tipo estado da arte que tem por objetivo identificar, descrever e sistematizar o que é denominado interdisciplinaridade e como ela se faz presente em trabalhos publicados nos anais de três edições da Conferência Nacional sobre Modelagem na Educação Matemática (CNMEM), ocorridas, respectivamente, nos anos de 2013, 2015 e 2017. Para melhor compreender essa pesquisa, apresentamos concepções das teorias que a guiam: modelagem matemática e interdisciplinaridade; e apresentamos os critérios utilizados para selecionar os textos analisados. Neste artigo, descrevemos quatro dos trabalhos eleitos a partir dos procedimentos metodológicos e realizamos suas análises. Do estudo, concluímos que é privilegiado, nos textos analisados, relatar as atividades que se caracterizam como interdisciplinares, em detrimento de discutir teoricamente essa teoria educacional no ensino de matemática.
\end{abstract}

Palavras-chave: Modelagem matemática na educação matemática. Interdisciplinaridade. Estado da Arte. CNMEM.

Thaís Ribeiro da Silva Aluna da Licenciatura em Matemática da Universidade Federal de Minas Gerais (UFMG).

iD https://orcid.org/0000-0002-7301-0993 $\bowtie$ thaisr1@ufmg.br

Paloma Silva de Lima Aluna da Licenciatura em Matemática da Universidade Federal de Minas Gerais (UFMG).

iD https://orcid.org/0000-0001-8661-6495 $\triangle$ palomasl@ufmg.br

Bárbara de Paula Motta Mirson Aluna da Licenciatura em Matemática da Universidade Federal de Minas Gerais (UFMG).

iD https://orcid.org/0000-0003-0552-4316 $\triangle$ barbaramirson@ufmg.br

Jussara de Loiola Araújo Doutorado em educação matemática pela Universidade Estadual Paulista (Unesp), campus Rio Claro. Professora da Universidade Federal de Minas Gerais (UFMG), Belo Horizonte, Minas Gerais, Brasil.

iD https://orcid.org/0000-0002-9156-2417 $\bowtie$ jussara@mat.ufmg.br

Recebido em 03/03/2021 Aceito em 22/03/2021 Publicado em 27/04/2021

\section{Interdisciplinarity in papers at the Conferência Nacional sobre Modelagem na Educação Matemática}

Abstract: In this paper, we present partial results of a state-of-the-art study that aims to identify, describe and systematize what is called interdisciplinarity and how it presents itself in published papers in the proceedings of three editions of Conferência Nacional sobre Modelagem na Educação Matemática (CNMEM), which occurred, respectively, in the years of 2013, 2015 and 2017. We present conceptions of the theories that guide the study - mathematical modelling and interdisciplinarity - and describe the criteria used to select the texts to be analysed. Here, we present and analyse four of the papers chosen from the methodological procedures. From the study, we conclude that, in the analysed papers, it is privileged to describe activities that are characterized as interdisciplinary, in detriment of theoretically discussing this educational theory in mathematics education.

Keywords: Mathematical modelling in mathematics education. Interdisciplinarity. State-of-the-art. CNMEM.

\section{Interdisciplinariedad en los trabajos en la Conferência Nacional sobre Modelagem na Educação Matemática}

Resumen: En este artículo presentamos resultados parciales de un estudio de tipo estado del arte que tiene como objetivo identificar, describir y sistematizar lo que se denomina por interdisciplinariedad y cómo está presente en trabajos publicados en las memorias de tres ediciones de la Conferência Nacional sobre Modelagem na Educação Matemática (CNMEM), ocurridos, respectivamente, en los años 2013, 2015 y 2017. Para comprender mejor esta 
investigación, exponemos concepciones de las teorías que la orientan: modelación matemática e interdisciplinariedad; y presentamos los criterios utilizados para seleccionar los textos analizados. En este artículo, describimos cuatro de los trabajos elegidos a partir de los procedimientos metodológicos. Concluimos que es privilegiado, en los textos analizados, relatar las actividades que se caracterizan como interdisciplinarias, en detrimento de discutir teóricamente esta teoría educativa en la enseñanza de matemáticas.

Palabras clave: Modelación matemática en educación matemática. Interdisciplinariedad. Estado del Arte. CNMEM.

\section{Introdução}

A modelagem matemática ${ }^{1}$ é uma importante tendência da educação matemática (BLUM et al., 2007; MEYER; CALDEIRA e MALHEIROS, 2011). Segundo Barbosa (2004), a modelagem matemática na educação matemática pode ser caracterizada com um "ambiente de aprendizagem" (p. 3), no qual os alunos buscam, por meio da matemática, investigar problematizações advindas de situações do cotidiano. Há diversas maneiras de organizar atividades de modelagem em ambientes escolares e, dependendo do principal propósito do professor, a atividade pode assumir diferentes perspectivas (KAISER e SRIRAMAN, 2006). No Brasil, as perspectivas mais usuais são a educacional (ALMEIDA; SILVA e VERTUAN, 2012; BASSANEZI, 2002), que entende a modelagem como um meio para ensinar matemática, e a sócio-crítica (ARAÚJO, 2012; ROCHA e PINTO, 2020), que a vê como uma possibilidade de realizar discussões críticas sobre o papel da matemática na sociedade. Por possuir caráter inovador frente ao ensino tradicional, a modelagem vem ganhando força e destaque na educação matemática brasileira por meio de práticas realizadas em sala de aula, pesquisas a respeito do tema, eventos destinados a discussões sobre essa tendência, dentre outros.

As discussões sobre esse campo no Brasil são amparadas em primazia pela Conferência Nacional sobre Modelagem na Educação Matemática (CNMEM), organizada pelo Grupo de Trabalho "Modelagem Matemática" (GT10) da Sociedade Brasileira de Educação Matemática, que congrega docentes de todos os níveis educacionais, pesquisadores e discentes que pesquisam, praticam e/ou são interessados pela modelagem (SBEM, 2019). Esse evento é realizado em diferentes instituições do país a cada dois anos desde 1999.

Por ser caracterizado como um espaço de encontro abrangente, a CNMEM inclui em sua programação relatos de experiências de professores que ensinam matemática e realizam práticas de modelagem, apresentações de pesquisadores que, por meio de comunicações científicas e relatos de experiência, divulgam suas pesquisas concluídas ou em andamento, além de palestras, debates

\footnotetext{
1 Para efeito de fluência do texto, em alguns momentos iremos nos referir à tendência educacional modelagem matemática na educação matemática como "modelagem matemática", "modelagem na educação matemática" ou "modelagem".
} 
temáticos e minicursos. Nesse sentido, os anais da conferência são ricos em trabalhos que refletem a diversidade de experiências relacionadas à modelagem que são vivenciadas no Brasil.

Tendo em vista esse contexto, apresentamos, neste artigo, resultados parciais de nossa pesquisa de iniciação científica². Nosso objetivo é identificar, descrever e sistematizar o que é denominado interdisciplinaridade e como ela se faz presente em trabalhos publicados nos anais da oitava, nona e décima edições da CNMEM realizadas, respectivamente, nos anos de 2013, 2015 e 2017. Escolhemos essas três edições porque a pesquisa visa avaliar trabalhos publicados a partir de 2012. Além disso, a CNMEM de 2019 não foi incluída devido ao fato de a pesquisa ter se iniciado antes de sua realização. Dessa forma, o recorte apresentado no presente texto é destinado a compartilhar resultados referentes à primeira fase de nosso estudo.

Para nós, a evidente presença de conteúdos que poderiam ser relacionados a outras disciplinas, além da matemática, em uma atividade de modelagem, e as concepções de modelagem e interdisciplinaridade, que serão abordados em seguida, justificam a pertinência de buscar compreender como a interdisciplinaridade se faz presente em atividades e pesquisas sobre modelagem, quando esse conceito é mencionado em trabalhos que descrevem tais atividades. Realizamos a pesquisa, então, a fim de entender mais a fundo como se dá a relação entre esses dois aportes teóricos na perspectiva da educação matemática.

Para que seja possível a familiarização do leitor com nosso objeto de estudo, começamos por apresentar, na próxima seção, uma discussão sobre modelagem na educação matemática e interdisciplinaridade, a fim de esclarecer algumas ideias teóricas. Isso será feito de forma concisa, visando introduzir as concepções teóricas exploradas em nossa pesquisa. Na seção 3 descrevemos os procedimentos metodológicos adotados na realização do estudo. Nossas análises e os respectivos resultados são exibidos na seção 4 e, então, apresentamos nossas considerações finais.

\section{Modelagem na educação matemática e interdisciplinaridade}

Inspirada no campo da matemática aplicada (BASSANEZI, 2002), mas construindo sua própria identidade na educação matemática (BARBOSA, 2004), a modelagem comumente é apresentada, na

\footnotetext{
2 A pesquisa de iniciação científica contou com o apoio do Programa Institucional de Bolsas de Iniciação Científica da PróReitoria de Pesquisa da UFMG, patrocinado pelo Conselho Nacional de Desenvolvimento Científico e Tecnológico (PIBIC/CNPq). Ela é intitulada "Ensino e aprendizagem na educação matemática: estado da arte relativo a aspectos interdisciplinares" e visa compreender como a interdisciplinaridade vem sendo abordada nos trabalhos de modelagem na educação matemática na América do Sul. Esse estudo compõe o levantamento realizado pelo Survey Team 4: On the teaching and learning of mathematical modelling and interdisciplinary mathematics education, que será apresentado no 14th International Congress on Mathematical Education (ICME-14) em Shanghai, China, em julho de 2021.
} 
educação matemática, por meio de problemas (escolhidos pelo docente e/ou pelos próprios estudantes) emergentes de situações cotidianas ou de outras áreas do conhecimento que são resolvidos com o apoio de conteúdos matemáticos que os estudantes já conhecem, necessitam ou que desejam aprender. Embora essa compreensão seja muito ampla e, portanto, não transmita várias das diferentes formas que a modelagem pode assumir na educação matemática (ALMEIDA; ARAÚJO e BISOGNIN, 2011; KAISER e SRIRAMAN, 2006), ela traduz características comuns à maioria dessas formas. Apresentaremos nessa seção, a fim de situar o leitor, algumas concepções da modelagem matemática na educação matemática, entretanto, ressaltamos que não é nossa intenção definir uma única perspectiva de modelagem que assumimos ao realizar a pesquisa, já que em seu decorrer buscamos considerar válidas quaisquer daquelas adotadas pelos autores dos textos que analisamos.

Um modelo, de acordo com Bassanezi (2002), pode ser entendido como um sistema artificial por meio do qual se formalizam argumentos ou parâmetros essenciais de uma porção da realidade. Para Blum e Borromeo Ferri (2009), em uma atividade de modelagem matemática há dois tipos de modelo: 0 modelo real e o modelo matemático. 0 modelo real aparece quando a situação modelo é sistematizada e estruturada. 0 modelo matemático é obtido a partir do modelo real, por meio do processo definido pelos autores como matematização, que pode ser representada, por exemplo, por equações que descrevem matematicamente a situação-problema estudada. Desse modo, o processo de construção de um modelo matemático passa a ser interpretado como a construção de um sistema artificial que se utiliza de argumentos matemáticos, elaborado na tentativa de representar uma porção da realidade, uma situação.

Cabe ressaltar que a finalidade última dos modelos não é sempre a mesma. Essas representações podem ter variados objetivos entre os quais estão prever o comportamento de um fenômeno, demonstrar um evento ou auxiliar pedagogicamente alguma compreensão (ALMEIDA; SILVA e VERTUAN, 2012). Dessa forma, na modelagem matemática os modelos têm, em geral, a função de auxiliar na resolução de um problema. Ademais, sua elaboração constitui uma atividade pedagógica para aqueles que a fazem.

Almeida, Silva e Vertuan (2012) apontam que uma atividade de modelagem matemática pode ser entendida como a busca de soluções para um problema - relativo a um tema não necessariamente matemático - com o apoio de modelos matemáticos. A essa atividade, os autores atribuem um método próprio constituído de etapas que levam um problema em situação inicial a uma situação final, que constitui uma solução para o problema. Dessa forma, entendem que os procedimentos utilizados na modelagem matemática são interação, matematização, resolução e interpretação de resultados $e$ validação que não ocorrem necessariamente nessa ordem, ou mesmo de forma sequencial. A interação corresponde, como sugere a própria palavra, ao ato de se inteirar da situação, entender sobre o tema de 
estudo e captar as informações sobre ele. Durante a matematização, semelhante ao que descrevem Blum e Borromeo Ferri (2009), é feita uma tradução dos aspectos considerados relevantes para constituir o modelo do problema em linguagem matemática. Já a resolução pode ser entendida como o momento no qual um modelo matemático que descreva a situação-problema é formulado e utilizado para analisála e responder às perguntas do problema. Finalmente, a interpretação de resultados e validação é o procedimento no qual os envolvidos na atividade avaliam as respostas indicadas pelo modelo e sua adequação ao problema. 0 desenvolvimento de uma atividade de modelagem é ilustrado na Figura 1:

Figura 1: Etapas de modelagem matemática

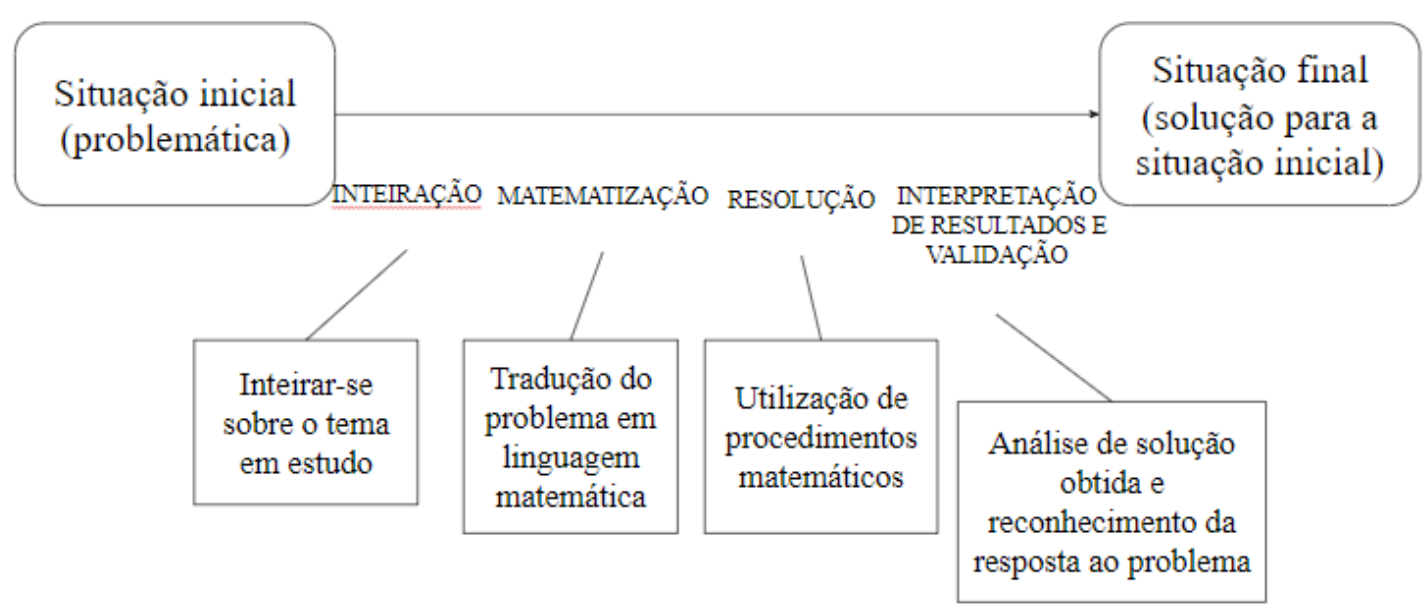

Fonte: Adaptado de Almeida, Silva e Vertuan (2012) por Veronez e Veleda (2016, p. 1241)

Um exemplo do uso da modelagem no âmbito da sala de aula é apresentado por Araújo (2012). Na situação descrita no artigo, um grupo de estudantes do curso de Geografia realizou um projeto de modelagem matemática, enquanto alunos da disciplina denominada Matemática. A autora destaca que o projeto, cujo tema foi "Aspectos socioeconômicos do projeto de construção da Linha Verde 3 em Belo Horizonte", tinha como objetivo relacionar o tráfego, a necessidade da construção de um viaduto e seus impactos na região em análise. Para isso, o grupo de estudantes calculou o fluxo de veículos na região; estimou, utilizando conceitos da engenharia, sua evolução durante os anos; e entrevistou moradores da área e pessoas que costumavam transitar a pé na região onde as obras da Linha Verde ocorreriam. Dessa forma, realizou-se um estudo que mobilizou conhecimentos das áreas de matemática, de geografia e de engenharia. Assim, cabe observar que esse trabalho, entre cujos objetivos não estava a intenção de possuir caráter interdisciplinar, evidencia o vínculo natural que atividades de modelagem podem promover entre a matemática e outras ciências.

\footnotetext{
${ }^{3}$ Linha Verde foi um projeto lançado em 2005 pelo governo de Minas Gerais que intencionou reestruturar vias da capital mineira, Belo Horizonte, e das cidades pertencentes à sua região metropolitana para melhorar as condições de tráfego nessas regiões.
} 
Ao realizar práticas, que envolvem situações do cotidiano do aluno ou de outras áreas do conhecimento, parece ser natural, então, que a matemática se relacione com outras disciplinas em atividades ou pesquisas sobre modelagem matemática, o que nos leva à ideia de interdisciplinaridade (MALHEIROS, 2012). Quando se aborda assuntos não matemáticos em uma atividade de modelagem, há a necessidade de compreender sobre esses assuntos para poder realizar a prática, já que o propósito da atividade é investigar a situação-problema proposta, utilizando ferramentas matemáticas. Dessa forma, percebe-se que a interdisciplinaridade pode cooperar no entendimento de assuntos novos ou daqueles já abordados, sendo capaz de estabelecer uma forte relação com a modelagem na educação matemática. Para Klüber (2012), o ensino de matemática por meio de modelagem matemática, assim como o de qualquer ciência, é de natureza interdisciplinar, mas o autor acrescenta que faz-se necessário distinguir as características interdisciplinares, presentes no cerne de uma disciplina, da interdisciplinaridade que é externa à essência dessa ciência. Ele pontua ainda que a natureza e a qualidade dessa interdisciplinaridade precisam ser bem pensadas para serem bem executadas, visando não reproduzir o ensino tradicional presente nas salas de aula.

Em sua concepção mais genérica, a interdisciplinaridade pode ser entendida como o uso de duas ou mais disciplinas escolares em alguma tarefa educacional (FAZENDA, 2008). Ivani Fazenda, entretanto, pontua que essa concepção, por ser muito ampla, não é capaz de definir todas as singularidades presentes no campo. Já Domingues (2011) define que a interdisciplinaridade e seus correlatos, como a multi e a transdisciplinaridade 4 , são tentativas de associar diferentes campos disciplinares e promover a fusão entre eles. Ele também conceitua a interdisciplinaridade, em conformidade com a Organização para a Cooperação e o Desenvolvimento Econômico, como a cooperação das disciplinas, visando uma linguagem própria, priorizando o método científico utilizado por cada uma e tendo, consequentemente, desdobramentos tanto no ensino quanto na pesquisa das ciências envolvidas.

No contexto escolar, a interdisciplinaridade comumente é baseada nas disciplinas presentes no currículo, mas pode não se restringir somente a elas (GARCIA, 2012), o que pode ser ilustrado pela prática apresentada em Araújo (2012) em que a engenharia se juntou à matemática e à geografia no desenvolvimento do projeto de modelagem. Assim, para Garcia (2012), por meio de um currículo amplo, que dialoga com a sociedade e com práticas investigativas, a interdisciplinaridade tem poder de atravessar as fronteiras da sala de aula e da própria escola. Nesse sentido, a interdisciplinaridade no

\footnotetext{
${ }^{4}$ Como não são foco deste estudo, não nos aprofundaremos nas discussões sobre as temáticas multi e transdisciplinaridade, mas ressaltamos que existem diferenças metodológicas entre essas três concepções. Portanto, elas não podem ser consideradas idênticas.
} 
ambiente escolar pode ir além de uma junção de conteúdos, tornando possível integrar conhecimentos advindos de pesquisas e experiências, explorando determinados assuntos, problemas ou questões sociais (GARCIA, 2012).

Mesmo que o caráter interdisciplinar seja atribuído a vários trabalhos de modelagem, a compreensão acerca desse conceito ainda é diversa entre quem o utiliza. Como também apontam Setti e Vertuan (2016), percebemos que, apesar de a ideia mais ampla de interdisciplinaridade estar atrelada a uma integração entre disciplinas, a intensidade e a forma dessa integração variam entre os entendimentos do termo. Evidenciamos, assim, que o universo que abrange os conceitos de interdisciplinaridade é vasto, embora seja um termo comumente atrelado à modelagem matemática.

Tendo em conta a diversidade de compreensões, tanto da modelagem quanto da interdisciplinaridade e da relação entre elas, se justifica, então, buscar compreender o que é denominado interdisciplinaridade e como ela se faz presente em trabalhos sobre modelagem na educação matemática, em particular na Conferência Nacional sobre Modelagem na Educação Matemática. Nesse sentido, nosso próximo passo é descrever os procedimentos metodológicos utilizados na busca e análise das comunicações científicas e relatos de experiência publicados nos anais das três edições da CNMEM escolhidas.

\section{Procedimentos Metodológicos}

A pesquisa, cujo recorte é aqui relatado, é do tipo estado da arte, que, segundo Fiorentini e Lorenzato (2006) é uma modalidade de pesquisa (histórico-)bibliográfica ou de revisão. 0 principal material empírico utilizado em tais pesquisas são documentos escritos e elas visam inventariar e sistematizar o conhecimento produzido por determinado campo em um período de tempo específico.

Na mesma linha desses autores, Ferreira (2002) afirma que as pesquisas do tipo estado da arte são de natureza bibliográfica e se caracterizam, principalmente, por realizarem inventários e descrições da produção científica e acadêmica. Em comum, continua a autora, essas pesquisas têm:

[...] o desafio de mapear e de discutir uma certa produção acadêmica em diferentes campos do conhecimento, tentando responder que aspectos e dimensões vêm sendo destacados e privilegiados em diferentes épocas e lugares, de que formas e em que condições têm sido produzidas certas dissertações de mestrado, teses de doutorado, publicações em periódicos e comunicações em anais de congressos e de seminários. (FERREIRA, 2002, p. 258).

Ressaltamos que neste artigo, apresentamos os resultados parciais de uma pesquisa mais ampla, que busca em diferentes fontes e espaços temporais, entender como se dá a relação entre modelagem na educação matemática e interdisciplinaridade na América do Sul. 
Em termos de análise, nosso ponto de partida foi um conjunto de 235 trabalhos publicados nos anais da oitava, da nona e da décima edições da CNMEM e, portanto, todos pertencentes ao campo da modelagem na educação matemática. Partindo de nossos estudos teóricos sobre interdisciplinaridade e em sintonia com design emergente de uma pesquisa (LINCOLN e GUBA, 1985), estabelecemos, então, três critérios para selecionar, desse conjunto, os trabalhos que seriam incluídos no corpus de nosso estudo:

1. Trabalhos que mencionem a palavra interdisciplinaridade e seus correlatos ao longo do texto;

2. Trabalhos que relatem alguma atividade prática de modelagem na qual duas ou mais ciências ou disciplinas atuam simultaneamente, sendo uma delas a matemática;

3. Trabalhos que discutem teoricamente relações entre matemática e outras ciências ou disciplinas.

Dos 235 trabalhos publicados, considerando os critérios descritos acima, selecionamos, inicialmente, 88 trabalhos que atendiam a pelo menos um deles para serem analisados. Entretanto, um dos obstáculos encontrados no percurso da pesquisa foi que, no começo da análise, percebemos que a maioria desses textos não considerava efetivamente aspectos interdisciplinares em seu conteúdo. Eles apenas mencionavam que a modelagem poderia promover atividades interdisciplinares, ou faziam afirmações semelhantes a essa, sem realizar uma discussão mais detalhada. Decidimos, então, realizar uma segunda seleção, visando encontrar textos em que houvesse essa discussão mais refinada. Buscamos em meio aos 88 trabalhos da CNMEM, anteriormente selecionados, aqueles que realizavam discussões mais substanciais sobre interdisciplinaridade. Para isso, fizemos um quadro de classificação dos artigos em que separamos os trabalhos de acordo com o nível de profundidade 5 da discussão do tema interdisciplinaridade. Nesse processo, selecionamos aqueles em que avaliamos haver maior dedicação ao teorizar sobre interdisciplinaridade. Ao final desta segunda seleção, listamos um total de 28 trabalhos para serem analisados.

Foi por meio deste estudo dos textos da lista final, com 28 trabalhos, que elaboramos nossas conclusões sobre a maneira como tem se dado a relação entre modelagem e interdisciplinaridade nessas edições da Conferência Nacional sobre Modelagem na Educação Matemática. Desse modo, analisando informações específicas de cada trabalho, o que detalharemos no início da quarta seção do presente texto, pudemos realizar uma análise mais objetiva sobre como é trabalhada a relação estudada em nossa pesquisa. Essas informações estão sintetizadas e organizadas por edição da CNMEM na Tabela 1 a seguir:

\footnotetext{
${ }^{5}$ Definimos como profundos os textos que discutem teoricamente ou apresentam conclusões sobre a interdisciplinaridade.
} 


\begin{tabular}{ccccc}
\hline $\begin{array}{c}\text { Edição da } \\
\text { CNMEM }\end{array}$ & Ano & $\begin{array}{c}\text { Total de trabalhos } \\
\text { publicados nos } \\
\text { anais }\end{array}$ & $\begin{array}{c}\text { Número de } \\
\text { trabalhos na } \\
\text { primeira seleção }\end{array}$ & $\begin{array}{c}\text { Número de } \\
\text { trabalhos na } \\
\text { segunda seleção }\end{array}$ \\
\hline VIII & 2013 & 79 & 39 & 10 \\
\hline IX & 2015 & 74 & 22 & 10 \\
\hline$X$ & 2017 & 82 & 27 & 8 \\
\hline & Total $\rightarrow$ & 235 & 88 & 28 \\
\hline
\end{tabular}

\section{A análise dos textos selecionados}

Para analisar cada trabalho, buscamos, em cada um dos textos, as seguintes informações: objetivo do trabalho, concepção de interdisciplinaridade adotada, resumo da prática de modelagem realizada, principais resultados e conclusões diretamente relacionadas à interdisciplinaridade. Dessa forma, acreditamos ter selecionado uma amostra a partir da qual pudéssemos melhor sistematizar 0 conhecimento relativo à presença de interdisciplinaridade em trabalhos de modelagem matemática nas três edições da CNMEM.

Nesta seção, escolhemos 4, dos 28 trabalhos obtidos na segunda seleção, a fim de ilustrar como foram realizadas essas análises. Optamos por apresentar 4 trabalhos, presentes nas edições de 2013, 2015 e 2017, porque são aqueles que se destacaram em nossas análises por possuírem maior discussão teórica acerca de interdisciplinaridade e por apresentarem conclusões mais substanciais sobre interdisciplinaridade.

\subsection{Descrição dos trabalhos}

O primeiro trabalho analisado é intitulado Modelagem matemática e sensores de temperatura na escola técnica, de Matté e Santana (2013). Esse relato de experiência tem como objetivo dar aos alunos condições para que percebam a importância de coletar e tratar dados, empregar conhecimentos matemáticos para interpretar resultados da atividade, além de proporcionar uma maior afinidade com conceitos de eletricidade. Há, também, a intenção de criar um ambiente favorável para que os alunos participem de discussões e, assim, haja uma construção mais dinâmica do conhecimento. Os autores consideraram a atividade interdisciplinar por esta abordar conteúdos da eletricidade, física e matemática, e, assim, promover uma integração entre conhecimentos de áreas diferentes. 
A atividade teve como contexto um curso técnico em Mecatrônica em uma Escola Técnica e consistia em, por meio de medições, construções de gráficos, observações e do trabalho em equipe, encontrar uma função matemática que descrevesse o comportamento dos termo-sensores estudados. Foi feita uma revisão de conceitos matemáticos que seriam amplamente utilizados: funções afim, logarítmicas e exponenciais. Após construírem as curvas relacionadas aos sensores, houve a validação dos resultados mediante uma conversa com a turma.

Os autores concluíram que a atividade aplicada mostrou aos alunos a realidade de que os resultados podem não ser exatos mesmo com o uso de matemática. Além disso, eles apontam que a modelagem matemática é potencializadora da aprendizagem dos estudantes e possibilitadora da revisão de conteúdos, além de propiciar a visão de que a matemática é útil na sociedade. No que se refere à interdisciplinaridade, concluem que, além de facilitar o aprendizado dos alunos, uma atividade interdisciplinar permite unir mais de uma área do conhecimento para que não haja a fragmentação do ensino em disciplinas fechadas, com pouca ou nenhuma relação com o cotidiano do aluno.

O segundo texto do qual apresentaremos a análise, cujo título é $A$ modelagem matemática no ensino de funções como possibilidade de reflexão para o consumo consciente, é de autoria de Mello (2013) e tem como objetivo criar intencionalmente situações de ensino e de aprendizagem na matemática visando promover diversas reflexões sobre as questões sociais relacionadas ao consumo consciente. Tem, também, foco em desenvolver o conceito de função por meio da modelagem matemática, construir tabelas, trabalhar com a representação gráficas de uma função linear e discutir sobre preços dos combustíveis e seus benefícios e prejuízos ao meio ambiente.

A atividade de modelagem matemática, que foi realizada em uma turma de Ensino Médio de uma Escola Técnica, contou com a participação de professores de várias disciplinas, o que a caracterizou como um projeto interdisciplinar. $O$ tema proposto era consumo consciente. As atividades realizadas em sala iniciaram-se com a proposição de uma lista de perguntas para os alunos. Eles discutiram as questões propostas pela professora e, posteriormente, tiveram a oportunidade de investigar acerca dessas questões. Foram usados conhecimentos matemáticos para montar tabelas relacionando o preço pago pelo combustível com a quantidade de litros comprados e desenhar gráficos de funções. Os produtos das atividades foram expostos na escola.

A autora concluiu que a modelagem matemática contribui para a aprendizagem dos alunos ao motivá-los a encontrar uma solução para o problema proposto. Houve, também, uma reflexão sobre como a matemática tem o papel de formar cidadãos críticos. Em relação à interdisciplinaridade, entendeu-se que a possibilidade de trabalhar em conjunto proporcionou um maior entrosamento entre os professores e melhor comprometimento por parte dos alunos. 
O terceiro trabalho analisado, de Vieira e Thiel (2015), tem por título Matemática no basquetebol. Os objetivos declarados no texto foram aproximar a matemática da realidade e, com isso, facilitar a compreensão dos alunos e fazê-los perceber que a matemática está presente em nosso cotidiano. Nesse sentido, os autores optaram pela "interdisciplinaridade considerando sua característica de aproximar currículos, valorizando algumas disciplinas dentro de um mesmo contexto" (VIEIRA e THIEL, 2015, p. 2).

A prática realizada teve como finalidade estudar o basquete em uma perspectiva da matemática, da física e da educação física. A atividade foi executada em uma turma do Ensino Médio de uma Escola Técnica, a partir de questionamentos relacionados ao esporte, feitos pelos próprios alunos. Esses estudantes, divididos em grupos, tiveram o papel de encontrar um modelo matemático para responder às questões estimuladas nas aulas.

Vieira e Thiel (2015) descrevem o papel de cada uma das disciplinas na atividade. Da Física, foram abordados os seguintes conceitos: energia cinética, grandezas vetoriais e escalares, gravidade, velocidade escalar e impulso, dentre outros. Os conceitos da matemática foram perímetro e área de figuras planas, volumes de sólidos geométricos, ângulos, equações, funções, gráficos e análise combinatória. A educação física teve o papel de "tornar mais visíveis" (VIEIRA e THIEL, 2015, p. 2) os conceitos da física e da matemática, além de abordar conteúdos próprios, como postura corporal, posicionamento em quadra, regras do basquete e de convivência em grupo.

Os autores concluíram, de maneira geral, que o estudo de algo que agrada os estudantes pode impactar positivamente sua visão sobre a matemática. Tratando especificamente da interdisciplinaridade, Vieira e Thiel (2015) enfatizam que o trabalho conjunto de interdisciplinaridade, modelagem e esportes viabilizou o aprendizado de muitos conteúdos matemáticos e físicos.

Por fim, o quarto trabalho que analisamos tem por título Pilhas eletroquímicas: uma abordagem didática com modelagem matemática, e é de autoria de Schwertner, Wille e Vertuan (2017). O objetivo declarado pelos autores é apresentar "duas atividades de Modelagem Matemática, envolvendo a construção e experimentação de pilhas eletroquímicas, num contexto de interdisciplinaridade com Química." (SCHWERTNER; WILLE e VERTUAN, 2015, p. 1).

A atividade narrada no texto em questão é realizada como um trabalho da disciplina de Modelagem Matemática de um curso de Licenciatura em Matemática. Na tentativa de descobrir a durabilidade de dois tipos de pilhas eletroquímicas que investigam, elas são construídas e 0 comportamento da corrente elétrica que passa por elas é modelado. A atividade realizada é caracterizada como interdisciplinar, já que há duas disciplinas trabalhando simultaneamente, a matemática e a química. 
O trabalho contém uma seção em que modelagem e interdisciplinaridade são discutidas simultaneamente. Nela, os autores apresentam sua compreensão de modelagem e as vantagens de se utilizar essa tendência da educação matemática em sala de aula. Apresentam também a concepção de interdisciplinaridade de Hilton Japiassu, que a entende de uma forma semelhante à que apresentamos aqui, como o trabalho conjunto de diferentes disciplinas. Ao final dessa seção, Schwertner, Wille e Vertuan (2017) reconhecem que a interdisciplinaridade pode contribuir para a escolha de temas em atividades de modelagem matemática, para a solução dos problemas e para a construção do conhecimento, em geral.

A conclusão desse relato focaliza a interdisciplinaridade e sua relação com a modelagem, ressaltando a construção de relações entre conteúdos de diversas disciplinas; a importância do trabalho conjunto de professores de diferentes disciplinas; a consideração de conhecimentos prévios dos alunos para a prática e viabilização de aprendizagem. Por fim, os autores afirmam que:

[...] a Modelagem Matemática, no que tange à interdisciplinaridade, possibilita relacionar os conceitos estudados fora do âmbito das disciplinas às quais competem, criando conexões entre disciplinas distintas $e$ fortalecendo diferentes áreas do conhecimento. (SCHWERTNER; WILE e VERTUAN, 2017. p. 12)

Após a descrição dos quatro trabalhos, vamos agora realizar uma análise conjunta deles.

\subsection{Análise dos trabalhos}

Retomando o objetivo deste estudo, já identificamos e descrevemos, na seção anterior, o uso que foi feito de interdisciplinaridade nos quatro trabalhos publicados nos anais de três edições da CNMEM. Passando para o momento de sistematização sobre o que é denominado interdisciplinaridade, constatamos que, nos quatro trabalhos, ela é entendida como o uso de duas ou mais disciplinas escolares, simultaneamente, em alguma tarefa educacional. Portanto, todos os trabalhos trazem a mesma compreensão inicial de interdisciplinaridade, como aquela descrita e questionada por Fazenda (2008), e não avançam em termos de discussões teóricas sobre o tema, embora existam inúmeros estudos sobre ele (DOMINGUES, 2011).

Notamos que, na maioria dos trabalhos publicados nos anais da CNMEM, a interdisciplinaridade é citada no intuito de atribuir à atividade relatada a característica de conter o trabalho entre duas ou mais disciplinas. Durante a análise, não enxergamos uma abordagem teórica mais profunda sobre o tema e, dos 28 trabalhos examinados e que foram considerados profundos, apenas os 4 analisados na subseção anterior apresentam conclusões sobre a importância de a atividade de modelagem matemática ser interdisciplinar. Apesar de muitos textos não apresentarem uma fundamentação teórica a respeito da interdisciplinaridade, o campo de estudos sobre esse assunto é amplo, podendo ser fortemente 
explorado por práticas e pesquisas nesse contexto. Isto é ressaltado por Setti e Vertuan (2016) ao relatarem que, de modo geral, as práticas interdisciplinares podem se diferenciar em características e intensidade.

Percebemos, portanto, que, geralmente, a interdisciplinaridade não se apresenta como um dos principais objetos de estudo, mas sim como um facilitador na escolha do tema sobre o qual se realizará a modelagem, conforme a primeira característica pontuada por Setti e Vertuan (2016). Ou seja, o professor utiliza temas e conceitos de outras disciplinas como uma maneira de contextualizar conteúdos matemáticos. Desta forma, a teoria se faz em torno da modelagem e a interdisciplinaridade é deixada em segundo plano. Outra característica observada é que, durante a realização da atividade prática, o ensino da matemática prevalece em relação às outras disciplinas.

Além disso, em três dos quatro trabalhos analisados, as atividades práticas foram realizadas em Escolas Técnicas e, o quarto, em um curso de Licenciatura em Matemática. No caso das Escolas Técnicas, de certa forma, todos os autores buscavam estabelecer conexões entre as diferentes disciplinas que compõem os cursos nos quais as atividades foram realizadas. Essa conexão poderia se dar em termos dos conteúdos das disciplinas ou do trabalho em parceria entre os respectivos professores. Esse objetivo vai ao encontro da descrição feita por Melillo (2017) do contexto de Escolas Técnicas e, em particular, de preocupações de professores de matemática em estabelecer uma maior conexão de sua disciplina com as demais disciplinas dos cursos.

No caso do curso de Licenciatura em Matemática, destacamos o objetivo de proporcionar, aos futuros professores, a oportunidade de realizar uma atividade prática que envolve duas importantes tendências, uma da educação matemática e outra da educação. Percebemos que no Ensino Superior, especificamente para 0 curso de Matemática, a modelagem na educação matemática ganha destaque como concepção teórica a ser aprendida com intuito de ser implementada em sala de aula. Desse modo, práticas de modelagem no contexto da formação inicial de professores de matemática aparecem como uma estratégia pedagógica, na qual, ao realizar uma prática como aprendiz, o docente pode projetá-la em seu trabalho, como indica o estudo de Barbosa (2001).

Diante do que observamos, apesar de parecer existir uma relação natural entre a modelagem, cujos temas são oriundos da vida real, e a interdisciplinaridade, que tem potencialidade para tratar esses temas em diferentes disciplinas, não são comuns os trabalhos nos quais essa relação é equilibrada. Ou seja, durante a realização da atividade, nota-se que é priorizada a abordagem teórica de modelagem à abordagem teórica de interdisciplinaridade. 


\section{Considerações Finais}

Com o intuito de identificar, descrever e sistematizar o que é denominado interdisciplinaridade e como ela se faz presente em trabalhos publicados nos anais de três edições da CNMEM, apresentamos a análise de quatro trabalhos. A partir disso, detectamos pontos de convergência entre eles e, dentre eles, destacamos a utilização de concepções muito semelhantes de interdisciplinaridade, o fato de a discussão estar sempre atrelada a uma atividade prática e a realização dessas atividades práticas em escolas técnicas ou cursos de graduação.

$\mathrm{Na}$ expectativa de encontrar trabalhos em que houvesse relação entre modelagem matemática e interdisciplinaridade foi que designamos os critérios para a busca. Como essa pesquisa foi feita a partir de textos publicados na CNMEM, pressupusemos que buscar por marcas que indicassem apenas a presença da interdisciplinaridade seria suficiente, uma vez que os trabalhos publicados nos anais da conferência versam sobre modelagem matemática.

Ao realizar essa pesquisa, percebemos que, por mais que a interdisciplinaridade seja comumente citada nos trabalhos de modelagem matemática, ela não se faz presente teórica ou substancialmente na maioria dos textos da conferência. Nossos resultados ilustram essa realidade, já que, de um total de 235 textos publicados nos anais das CNMEM de 2013, 2015 e 2017, 88 citam a palavra interdisciplinaridade e seus correlatos, e destes, apenas 28 apresentaram um conteúdo teórico acerca dessa tendência. Apontamos, por isso, a necessidade de uma maior fundamentação teórica nos trabalhos apresentados na CNMEM, uma vez que as relações entre interdisciplinaridade e modelagem precisam ser bem pensadas para serem bem executadas (KLÜBER, 2012).

Por outro lado, se dos 235 textos, 88 citam a palavra, percebemos a importância dada pelos professores e pesquisadores a essa união, fazendo-se necessários mais estudos sobre o tema. Então fechada essa parte, demos continuidade à nossa pesquisa a fim de entender como essa relação ocorre na literatura da América do Sul. A partir dos anais vimos que deles pudemos elaborar resultados interessantes, que serão por nós apresentados em maior completude, conjuntamente com as conclusões que elaboramos a partir de outras fontes, ao final dessa pesquisa.

\section{Referências}

ALMEIDA, Lourdes M. W.; ARAÚJO, Jussara L.; BISOGNIN, Eleni (Orgs.). Práticas de modelagem na educação matemática: relatos de experiências e propostas pedagógicas. Londrina: Editora da Universidade Estadual de Londrina, 2011. 
ALMEIDA, Lourdes Werle de; SILVA, Karina Pessôa; VERTUAN, Rodolfo Eduardo. Modelagem matemática na educação básica. São Paulo: Editora Contexto, 2012.

ARAÚJO, Jussara L. Ser crítico em projetos de modelagem em uma perspectiva crítica de educação matemática. Bolema, Rio Claro, v. 26, n. 43, p. 839-859, ago. 2012. DOI: 10.1590/S0103636X2012000300005.

BARBOSA, Jonei C. Modelagem matemática e os professores: a questão da formação. Bolema, Rio Claro, v. 14, n. 15, p. 5-23, 2001. Disponível em: <https://www.periodicos.rc.biblioteca.unesp.br/index.php/bolema/article/view/10622>. Acesso em: 21 abr. 2021.

BARBOSA, Jonei C. Modelagem matemática: O que é? Por quê? Como? Veritati, Salvador, v. 3, n. 4, p. 73-80, jun. 2004.

BASSANEZI, Rodney C. Ensino-aprendizagem com modelagem matemática. São Paulo: Editora Contexto, 2002.

BLUM, Werner; GALBRAITH, Peter L.; HENN, Hans-Wolfgang; NISS, Mogens (Eds.). Modelling and applications in mathematics education: The 14th ICMI study. New York: Springer, 2007.

BLUM, Werner; BORROMEO FERRI, Rita. Mathematical modelling: Can it be taught and learnt? Journal of Mathematical Modelling and Application, Blumenau, v. 1, n. 1, p. 45-58, dez. 2009. Disponível em: <https://proxy.furb.br/ojs/index.php/modelling/article/view/1620>. Acesso em: 21 abr. 2021.

DOMINGUES, Ivan. Disciplinaridade, multi, inter e transdisciplinaridade - onde estamos? Instituto de Estudos Avançados Transdisciplinares (IEAT). Belo Horizonte: Universidade Federal de Minas Gerais, 2011. Disponível em: <https:/www.ufmg.br/ieat/2012/03/disciplinaridade-multi-inter-etransdisciplinaridade-\%E2\%80\%93-onde-estamos/>. Acesso em: 13 jan. 2021.

FAZENDA, Ivani C. A. In: FAZENDA, I. (Org.). 0 que é interdisciplinaridade? São Paulo: Cortez Editora, 2008. p. 17-28.

FERREIRA, Norma S. A. As pesquisas denominadas "estado da arte". Educação \& Sociedade, Campinas, v. 23, n. 79, p. 257-272, ago. 2002. DOI: 10.1590/S0101-73302002000300013.

FIORENTINI, Dario; LORENZATO, Sérgio. Investigação em educação matemática: percursos teóricos e metodológicos. Campinas: Autores Associados, 2006.

GARCIA, Joe. O futuro das práticas de interdisciplinaridade na escola. Revista Diálogo Educacional, Curitiba, v. 12, n. 35, p. 209-230, jul. 2012. DOI: 10.7213/dialogo.educ.5908.

KAISER, Gabriele; SRIRAMAN, Bharath. A global survey of international perspectives on modelling in mathematics education. The International Journal on Mathematics Education, v. 38, n. 3, p. 302-310, 2006. Disponível em: <http://subs.emis.de/journals/ZDM/zdm063a9.pdf>. Acesso em: 21 abr. 2021.

KLÜBER, Tiago. E. (Des)encontros entre a modelagem matemática na educação matemática e a formação de professores de matemática. Alexandria, Florianópolis, v. 5, n. 1, p. 63-84, mai. 2012. Disponível em: <https://periodicos.ufsc.br/index.php/alexandria/article/view/37697>. Acesso em: 21 abr. 2021.

LINCOLN, Y. S.; GUBA, E. G. Naturalistic inquiry. Califórnia: Sage Publications, Inc., 1985. 
MATTÉ, Israel; SANT'ANA, Marilaine F. Modelagem matemática e sensores de temperatura na escola técnica. In: CONFERÊNCIA NACIONAL SOBRE MODELAGEM NA EDUCAÇÃO MATEMÁTICA, 8., 2013, Santa Maria, RS. Anais... Santa Maria: Centro Universitário Franciscano, 2013.

MALHEIROS, Ana Paula S. Pesquisas em modelagem matemática e diferentes tendências em educação e em educação matemática. Bolema, Rio Claro, v. 26, n. 43 p. 861-882, ago. 2012. DOI: 10.1590/S0103$636 \times 2012000300006$.

MELILLO, Célio R. A dualidade na formação no ensino profissionalizante em um ambiente de aprendizagem de modelagem matemática. 2017. 227 f. Tese (Doutorado em Educação) - Faculdade de Educação, Universidade Federal de Minas Gerais, Belo Horizonte.

MELLO, Jéssica. A modelagem matemática no ensino de funções como possibilidade de reflexão para o consumo consciente. In: CONFERÊNCIA NACIONAL SOBRE MODELAGEM NA EDUCAÇÃO MATEMÁTICA, 8., 2013, Santa Maria, RS. Anais... Santa Maria: Centro Universitário Franciscano, 2013.

MEYER, João F. C. A.; CALDEIRA, Ademir D.; MALHEIROS, Ana Paula S. Modelagem em educação matemática. Belo Horizonte: Autêntica Editora, 2011.

ROCHA, André L.; PINTO, Márcia M. F. A modelagem matemática na educação como recurso na formação crítica dos alunos no Ensino Fundamental. Revemop, Ouro Preto, v. 2, p. e202017, 2020. DOI: 10.33532/revemop.e202017.

SBEM. XI Conferência Nacional Sobre Modelagem na Educação Matemática, 2019. Modelagem matemática na educação matemática e a escola brasileira: atualidades e perspectivas. Disponível em: <http://eventos.sbem.com.br/index.php/cnmem/2019>. Último acesso em: 16 nov. 2020

SETTI, Elenice J. K.; VERTUAN, Rodolfo E. Que interdisciplinaridade se verifica nos trabalhos de modelagem matemática? In: Encontro Paranaense de Modelagem na Educação Matemática, 7, 2016. Londrina: Anais... Londrina: SBEM, 2016.

SCHWERTNER, Anderson; WILLE, Jackson; VERTUAN, Rodolfo. Pilhas eletroquímicas: uma abordagem didática com modelagem matemática. In: CONFERÊNCIA NACIONAL SOBRE MODELAGEM NA EDUCAÇÃO MATEMÁTICA, 10., 2017, Maringá, PR. Anais... Maringá: Universidade Estadual de Maringá, 2017.

VERONEZ, Michele R. D.; VELEDA, Gabriele G. Reflexões sobre a realidade em uma atividade de modelagem matemática. Perspectivas da Educação Matemática, Campo Grande, v. 9, n. 21, p. 12371252, dez. 2016. Disponível em: <https://periodicos.ufms.br/index.php/pedmat/article/view/1927>. Acesso em: 21 abr. 2021.

VIEIRA, Grasiella; THIEL, Afrânio. A matemática no basquetebol. In: CONFERÊNCIA NACIONAL SOBRE MODELAGEM NA EDUCAÇÃO MATEMÁTICA, 9., 2015, São Carlos, SP. Anais... São Carlos: Universidade Federal de São Carlos, 2015. 